\title{
A!
}

This is an electronic reprint of the original article.

This reprint may differ from the original in pagination and typographic detail.

Melvasalo, Maarit; Koivunen, Visa

\section{Using Spectrum Maps for Surveillance Avoiding Path Planning}

\section{Published in:}

2018 IEEE 19th International Workshop on Signal Processing Advances in Wireless Communications, SPAWC 2018

DOI:

10.1109/SPAWC.2018.8446033

Published: $24 / 08 / 2018$

Document Version

Peer reviewed version

Please cite the original version:

Melvasalo, M., \& Koivunen, V. (2018). Using Spectrum Maps for Surveillance Avoiding Path Planning. In 2018 IEEE 19th International Workshop on Signal Processing Advances in Wireless Communications, SPAWC 2018 (Vol. 2018-June). [8446033] (IEEE International Workshop on Signal Processing Advances in Wireless Communications). IEEE. https://doi.org/10.1109/SPAWC.2018.8446033

This material is protected by copyright and other intellectual property rights, and duplication or sale of all or part of any of the repository collections is not permitted, except that material may be duplicated by you for your research use or educational purposes in electronic or print form. You must obtain permission for any other use. Electronic or print copies may not be offered, whether for sale or otherwise to anyone who is not an authorised user. 


\title{
Using Spectrum Maps for Surveillance Avoiding Path Planning
}

\author{
Maarit Melvasalo and Visa Koivunen \\ Department of Signal Processing and Acoustics \\ Aalto University, Espoo, Finland \\ Email: \{maarit.melvasalo, visa.koivunen\}@aalto.fi
}

\begin{abstract}
Radio frequency surveillance avoiding path planning for platforms such as aircrafts is becoming increasingly challenging due to dense spectrum use and evolving radar systems. In order to find a safe path we need to acquire and learn awareness about the total exposure to radars. In practice, surveillance by radars can not be totally avoided, but it must be minimized or kept in a tolerable level. In this paper we first construct spectrum maps which present the levels of adversary surveillance signals as well as unintentional interferences. These maps are used to create virtual potential fields where adversary surveillance signals are modeled as repulsive forces. The proposed path planning algorithm navigates through such a potential field. It is a heuristic multistep algorithm that plans a surveillance avoiding path that trades off between the path length and the total level of surveillance signals illuminating the aircraft during the mission.
\end{abstract}

\section{INTRODUCTION}

Crowded radio spectrum is becoming a serious problem as more and more radio systems are developed to operate in spectrum bands with favorable propagation properties. Due to limited number of such bands, different radio systems such as radar and communication system need to share the common spectrum, [1]. At the same time the radar systems are evolving towards more agile usage of passive radar systems, for example DVB-T based systems. This has motivated the surveillance avoiding path planning of aircrafts, since the amount of radar exposure has increased and locations of passive radar receivers are unknown.

Typically, the path planning aims to find the shortest possible path while avoiding obstacles. In the case of aircrafts the optimum path might not be the shortest. In addition to other aircrafts, radar exposure and other surveillance systems as well as severe weather conditions can be considered as obstacles that need to be avoided. These obstacles are not solid objects like in typical path planning where a collision free path is targeted. Finding the optimum path has been extensively studied in the literature especially in the field of robotics [2]. In [3] a survey for autonomous motion planning for UAVs is given and in [4]-[6] the problem of both minimizing the travel distance and the threat exposure has been addressed.

In this paper, we propose an approach to the path planning problem, where spectrum maps are constructed and utilized to present the threat of adversary radars and other radio frequency surveillance sensors. For now we have limited for now the study to $2 \mathrm{D}$ scenarios but the proposed approach could be easily extended to 3D. First, spectrum maps are constructed based on spectrum sensing measurements and they are further used to define potential fields with repulsive and attractive forces. Next, several different alternative paths are searched by varying the strength of the attractive force and grid size are searched. This stage of the algorithm can be categorized as a potential field guided search method, see [3]. Finally, the threat is evaluated along these candidate paths. The final path selection is left for the pilot, or alternatively it can automated with selected preferences such as the length of the path and the probability of being detected by the adversary surveillance system.

\section{SPECTRUM CARTOGRAPHY AND ESTIMATION}

In order to present the state of the radio spectrum and activity of adversary surveillance systems, spectrum cartography (SC) can be used. Spectrum maps are spatio-temporal maps presenting the strength of the radio frequency signals in different locations and frequencies at different time instances, see e.g. [7]-[10]. Spectrum maps are constructed using a distributed multisensor system and other available surveillance information. Spectrum sensing provides RF measurements in distinct locations. However one also needs to know the state of the spectrum between the sensor locations. The unit used in the measurements and spectral maps typically is RSSI, received signal strength indicator.

In [10] we introduced a novel algorithm to construct spectrum maps for co-existing communication and radar systems. It breaks the problem to static and dynamic parts in order to generate a more accurate spectrum map and capture the dynamic or periodic scanning patterns of radar systems. Fist, the static part of a spectral map is generated using spatial Kriging interpolation, see [10]. The interpolation is based a set of actual measured values at known locations and an estimate of the spatial structure of second order statistic, i.e. covariance or variogram. The unknown values at any other location are estimated as linear combinations of the measured values as follows:

$$
\hat{Z}(\mathbf{x}, \mathbf{y})=\sum_{i=1}^{K} \lambda(i) \hat{Z}\left(\mathbf{x}_{i}, \mathbf{y}_{i}\right) .
$$

here $\lambda(i)$ are the Kriging weights and $\hat{Z}\left(\mathbf{x}_{i}, \mathbf{y}_{i}\right)$ are the measurements at $K$ known locations. In this paper we used the universal Kriging method with trend model depending on the first order polynomial function of location. The weights, $\lambda(i)$ 
are derived jointly with the trend parameters $\beta$ by minimizing the estimation variance of the mean at known sensor positions. The unbiasedness of the estimator is obtained by imposing a constraint on the weights $\sum_{i} \lambda(i)=1$. For modeling the spatial correlations a spherical variogram model [8] is used: $\gamma(h)=\left[\frac{3}{2} \frac{h}{a}-\frac{1}{2}\left(\frac{h}{a}\right)^{3}\right]$, if $h<a$, where $h$ is the distance and $a$ is the range parameter. It defines the maximum distance where two values are still correlated.

Typically the radars are scanning periodically with narrow beams that adds a dynamic component to the spectrum map. For the pathfinding problem we consider the radar beams as static with a wide beamwidth covering the whole scanning sector. The contribution of the radar beams to the static spectrum map is hence the maximum:

$$
\mathbf{R}(\mathbf{x}, \mathbf{y}, \mathbf{f})=\hat{Z}(\mathbf{x}, \mathbf{y}, \mathbf{f})+\sum_{k} \max _{t \in T} \mathbf{R}_{\mathbf{k}}(\mathbf{x}, \mathbf{y}, \mathbf{f}, t),
$$

where $\mathbf{R}_{\mathbf{k}}(\mathbf{x}, \mathbf{y}, \mathbf{f}, t)$, is a spectral map for $k$ th radar in the frequency band $f$ and one time instant $t$.

\section{PATHFinding}

The path planning is subdivided into two stages: the candidate path finding stage and the analysis stage. In a path planning problem, one of the key decisions is to define how to present the spectral environment. In a hostile environment, this is even more challenging, since there are many uncertainties, including the locations of the radars and their other operational parameters. Furthermore, how to set the path planning objectives and constraints when it is known that there are no collision-free paths, i.e. the aircraft can not totally avoid all the threat zones. In this paper, we present the navigation environment as discrete time potential fields. The spectrum map is used as a basis of a potential field model employed in the path planning. Similar model has been employed for example, for scheduling and routing purposes in wireless communications [11].

\section{A. Virtual force field}

In the potential field methods a potential function is assigned to free space such that the desired destination point has the lowest potential and it hence attracts the platform. Correspondingly, the obstacles are defined as repulsive forces to the platform. A potential field can be constructed by combining attractive and repulsive forces, and it can also be called a virtual force field. The virtual force field is a weighted combination

$$
F_{\alpha}=\alpha F_{a}+F_{r},
$$

where $F_{a}$ and $F_{r}$ are the attractive and repulsive forces respectively. The weight $\alpha$ is a model parameter which can be varied. The attractive force, $F_{a}$, used in this paper is the commonly used square distance from the goal. It reflects the importance of achieving the goal position. The repulsive force is obtained from spectral maps described in previous section so that it reflects the strength of the adversary surveillance signals.
In path planning it is essential to know the spatial threat from all frequencies, hence we combined several spectral maps from different bands together. We have used the maximum combining of the RSSI values over the whole bandwidth, i.e :

$$
F_{r}=\max _{f \in B} \mathbf{R}(\mathbf{x}, \mathbf{y}, \mathbf{f}) .
$$

In case some frequencies are considered more important than others, weighting of the different spectral maps could be incorporated into the model by setting a frequency specific weight $w_{f}$ to each map $\mathbf{R}(\mathbf{x}, \mathbf{y}, \mathbf{f})$,.

In order to find different candidate paths, the scaling parameter $\alpha$ is varied in the first stage of the proposed planning algorithm. With large $\alpha$ the attractive force becomes more dominant and the repulsive forces caused by flying thru a radar surveillance area are less influential. By decreasing $\alpha$ the emphasize is in avoiding radar surveillance.

\section{B. Discrete path}

A well known limitation of the potential field methods is getting trapped in a local minima [3]. This is due to usage of gradient descent approach in the path planning. Therefore, alternative discrete guided methods have been used with the potential fields, see survey paper [3]. In this paper we use the classical Dijkstras search algorithm [2], since it always finds a path and does not get stuck to a local minima.

Unlike typical robotic pathfinding problems, we have used an uniformly sampled $(N \times N)$ grid and all the grid points can be accessed. Moving from one grid point is allow to its eight neighbors. Note that Dijksrtas algorithm can be used with any grid geometry, shape or dimension and the typical cost function is the length of the path. In this paper we use the virtual force field values defined earlier as the basis of the cost function is. In case of Manhattan type movements, where only four directions are allowed, the virtual force field values can be used as such to present the cost function. Since now the eight neighboring cells are potential new locations, the actual distance in diagonal directions, i.e. $\sqrt{2}$, needs also to be taken into account in the cost function. The total cost from moving from point $\left(x_{i}, y_{i}\right)$ to its neighbor $x_{i+m_{x}}, y_{i+m_{y}}$ is the weighted virtual force field value:

$$
C_{i m}=|m| F_{\alpha}\left(x_{i+m_{x}}, y_{i+m_{y}}\right) .
$$

Here $m_{x}$ and $m_{y}$ are $\in\{-1,0,1\}$. The actual weights $|m|$ are hence either 1 or $\sqrt{2}$. The total cost for each path from the start to the goal is the cumulative sum. The minimization problem can be formulated as: $\min _{\text {all paths }} \sum_{i=\text { start }}^{\text {goal }} C_{i m}$.

With Dijkstras algorithm the complexity of path planning might be an issue, if the used discrete grid is too big. However, we can select the number of grid points such that the complexity does not pose problems. Here we have integrated the spectrums maps with a dense grid size such that the distance between neighboring grid points corresponds to the distance an aircraft is able to fly in between two radar scans. Correspondingly the virtual force field, see equation (3), is calculated first with this dense grid. For the path planning the 
grid is reduced to for example $30 \times 30$ evenly spaced grid points. The force field values are calculated as median (or mean) of the values from the neighbors. The neighborhood is defined so that each dense grid point is mapped into the neighborhood of the closest sparse grid point.

\section{THREAT ANALYSIS}

In the second stage of our path finding algorithm, the risk for all the found candidate paths is evaluated and the path selection is performed. For this evaluation, we use the joint detection probability of all known radars. We assume for now that we know the receiver operation characteristic (ROC) curves, or that we have another sophisticated way of the mapping the observed signal strength to probability of detection.

For each found candidate path, we calculate the probability of detection along direct path in between the earlier found points on the path. The speed of the aircraft and the radar scanning cycle time are take into account when the calculating this final stage. The signal strength is calculated for each final path points according to the radar equation with aircrafts radar cross section (RCS). For illustration purposes, we assume that RCS is constant for the aircraft.

\section{Simulation Results}

In our experiments we have used measured data to construct the spectral maps for different frequencies. The measurements are from DVB-T network, see [12]. For the radars, we placed three radars with $90 \mathrm{deg}$ scanning sectors in the area. The transmitted power for all radars is $50 \mathrm{~dB}$ stronger than powers observed from the DVB-T measurements. At the initialization stage, spatial spectrum maps are interpolated with Universal Kriging interpolation method. We have used interpolation grid of $0.5 \mathrm{~km}$, based on assumption of aircraft velocity of $900 \mathrm{~km} / \mathrm{h}$ and radar scanning interval of 2 seconds. The radar location and scanning parameters are assumed to be known or estimated reliably so the spectral maps for radars can be calculated. The spectral maps combined according to equation (4) to present the total repulsive force.

In the first stage total of 16 candidate paths were searched with four different $\alpha$ values and four different grid sizes $(N \in[10,15,30,60])$. The sparse force field values were calculated as a median value of the neighbors. In Figure 1 is shown for one grid size $N=15$ all the paths with the four different attractive force selections, i.e. different values of $\alpha$. In the upper left corner, the figure it can be seen that if the attractive force is very strong the path is direct. By decreasing the attractive force the path starts to avoid the radar beams. If the attractive force is weak enough, a path avoiding almost all radar exposure is found, see lower right figure. In Figure 2 is shown the impact of the employed grid size while keeping $\alpha$ constant. In this case the median attractive and repulsive forces are set equal, and four different grid sizes are used. In this example, the path found with three smallest grid sizes $(N=[10,15,30])$ is roughly the same. In case of the finest grid $(N=60)$, an alternative path which goes in between two radar beams is found.
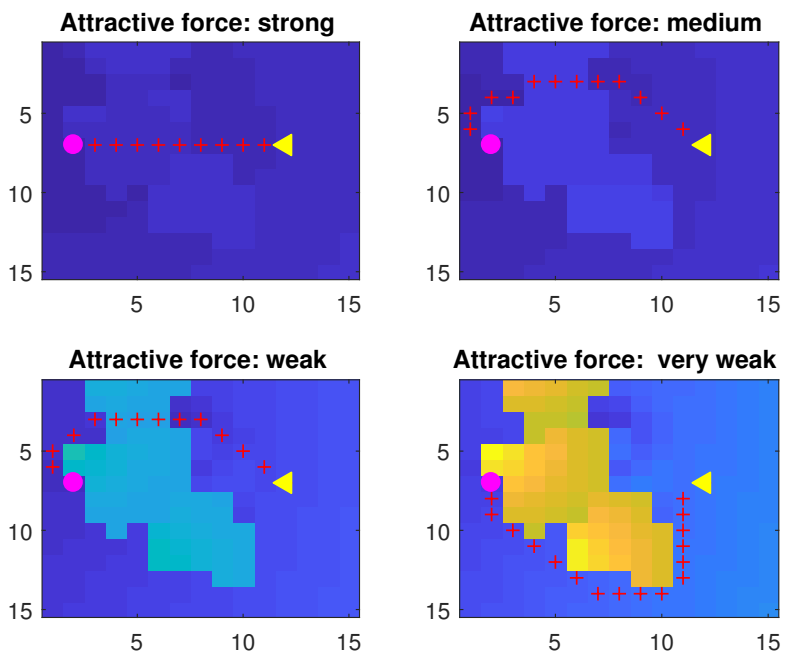

Fig. 1. Example of paths with four different virtual force fields and with grid size $15 \times 15$. Medium force refers to case where the median repulsive and attractive forces are equal. Strong attractive force has median two times the repulsive force. In case of weak or very weak attractive forces the ratio of the medians forces are $1 / 5$ and $1 / 10$.
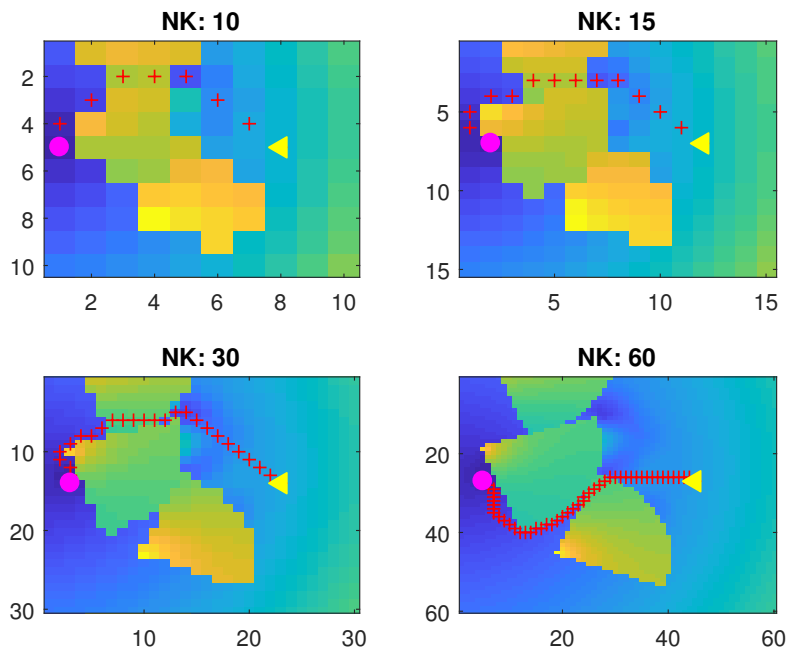

Fig. 2. Example of paths with four different grid sizes $N \in[10,15,30,60]$. In all plots the median of the repulsive and attractive forces are equal. This is referred here as medium attractive force.

The instantaneous probability of detection $(P D)$ along 16 different candidate paths are plotted in Figure 3 versus time index, which correspond to one radar scan, i.e. in our case every $0.5 \mathrm{~km}$. Consequently, if the number of radar scans along the path is 545 , the actual length of the path is $272.5 \mathrm{~km}$. In each plot the $P D$ is plotted for four different grid sizes. In the legend are shows the number of scan and the maximum instantaneous $P D$ on the path. The plot in the upper left corner shows $P D$ curves in the case of strong attractive force. In this case all the paths pass across radar surveillance zones, and the maximum $P D$ is $>0.9$. The curve for $N=15$ (red) is the shortest (408 radar scans), but on the other hand it has $P D>0.9$ for a long time (120 continuous scanning periods 

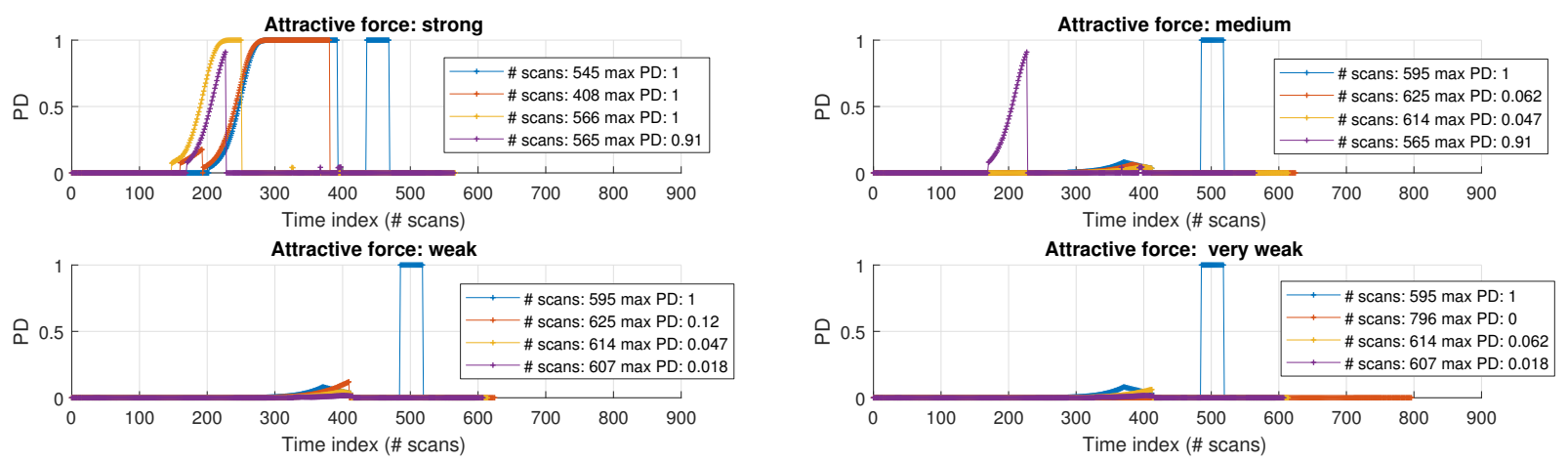

Fig. 3. Momentary probability of detection $(P D)$ versus the length of the path. In each plot, there are four paths found using different grid sizes. Blue curves correspond to $N=10$, red to $N=15$ and yellow to $N=30$. The purple curves are derived with $N=60$.
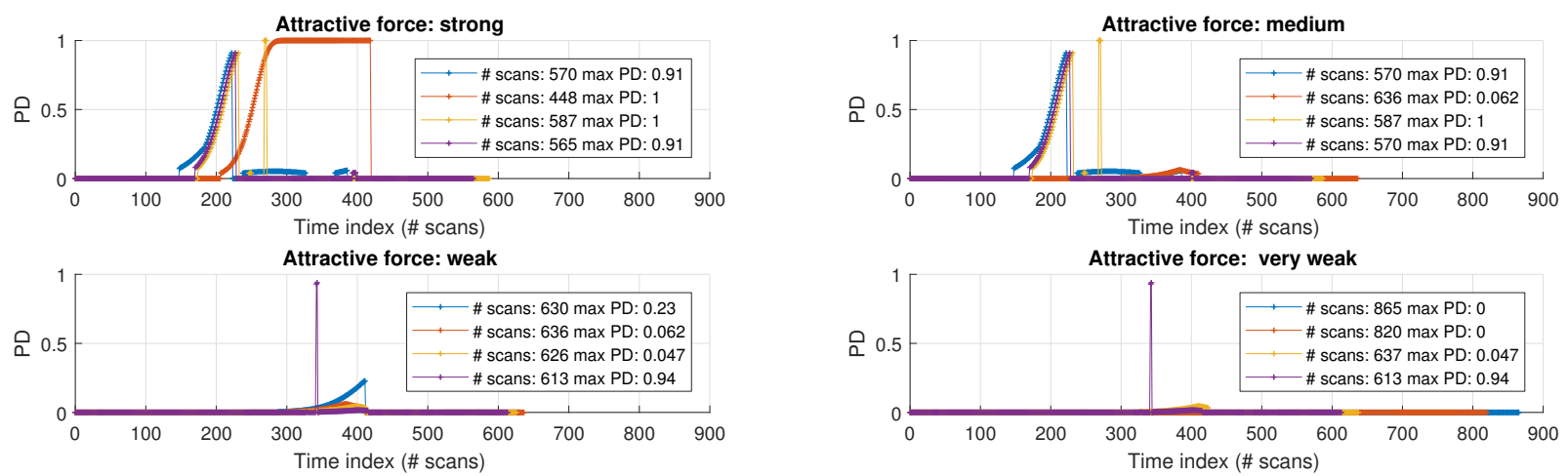

Fig. 4. Similar to Figure 3, but the force field values are obtained by using mean value instead of median value.

which indicates that a high quality radar track may be achieved by the adversary). With $N=10$ (blue) the situation is even worse. With $N=30$ (yellow curve) the PD is above 0.9 for 40 scanning periods, but with $N=60$ only one point is above this threshold.

In the other three plots of Figure 3, the instantaneous PDs are generally much lower, since the attractive force is weaker and the algorithm emphasizes avoidance of surveillance radars more. Most commonly the length of the found paths is about $300 \mathrm{~km}$ and the maximum instantaneous $P D$ is below 0.1 . This type of path can bee seen, for example, in Figure 2 with grid sizes $N=10,15,30$. In the same figure with $N=60$ the path is a bit shorter but the $P D$ is clearly higher. From Figure 3 we see, that all the blue curves have $P D=1$ for over 30 scans. This is due to the path cutting corners a bit too eagerly when the gird size is small, $N=10$. Out of the 16 candidate paths only one grid size has $\max P D=0$. This is the case with $N=15$ and very small $\alpha$, i.e. very weak attractive force. This route is, however, $100 \mathrm{~km}$ longer than any other route.

Pathfinding was also simulated using a mean-valued force field, i.e. a sparse grid was defined using the mean of the neighborhood values instead of the median. In Figure 4 are show the instantaneous $P D$ versus the length for the 16 different paths similarly to the Figure 3 . The first important observation here is that the cutting of corners with the small grid value is no longer a problem. However, in some cases the maximum instantaneous $P D$ has some strong peaks. Third observation is that now two paths have max $P D=0$.

\section{CONCLUSION}

In this paper we developed a method for planning paths for a platform such as aircraft in spectrally dense environment such that radar signals or interference can be avoided. Spectrum maps are constructed and surveillance signals are modeled as repulsive forces in a virtual potential field. The proposed path planning method is a two-stage algorithm. In the first stage a guided potential field method is used to find several candidate paths from a starting point to the destination point. The grid size and the relative ratio of the attractive and repulsive forces are altered to find different paths with different resolution. In the analysis stage the probability of detection is estimated along the candidate paths. The proposed method offers an automated way of finding paths that trade off between length and surveillance avoidance.

\section{ACKNOWLEDGMENT}

The authors would like to thank the Finnish Defense Forces for financial support and valuable comments to this work. 


\section{REFERENCES}

[1] Hugh Griffiths, Lawrence Cohen, Simon Watts, Eric Mokole, Chris Baker, Mike Wicks, Shannon Blunt, S, "Radar Spectrum Engineering and Management Technical and Regulatory Issues," Proceedings of the IEEE, vol. 103, no. 1, pp. 85-102, 2015.

[2] S. M. LaValle, Palnnin Algorithms. Cambridge University Press, 2006.

[3] C. Goerzen, Z. Kong, B. Mettler, "A Survey of Motion Planning Algorithms from the Perspective of Autonomous UAV Guidande," Journal of Intelligent and Robotics Systems, pp. 57-65, 2010. https: //doi.org/10.1007/s10846-009-9383-1.

[4] W.M. Carlyle, J.O. Royset, R. K. Wood, "Routing military aircraft with aconstrained shortest-path algorithm," Military Operations Research, vol. 14, no. 3, pp. 31-52, 2007.

[5] J. Berger, A. Boukhtouta, A. Benmoussa, O. Kettani, "A New mixedinteger linear programming model for rescue path planning in uncertain adversarial environment," Computers and Operational Research, vol. 39, pp. 3420-3430, 2012.

[6] Tina Erlandsson, "Route planning for air missions in hostile environments," Journal of Defence Modelling and Simulation: Application, Methology, Technology, 2015.

[7] A. B. H. Alaya-Feki, S. B. Jemaa, B. Sayrac, P. Houze and E. Moulines, "Informed spectrum usage in cognitive radio networks: Interference cartography," in 2008 IEEE 19th International Symposium on Personal, Indoor and Mobile Radio Communications, pp. 1-5, Sept 2008.

[8] Gabriele Boccolini, Gustavo Hernandez-Penaloza, Baltasar BeferullLozano, "Wireless Sensor Network for Spectrum Cartography Based on Kriging Interpolation," in International Symposium on Personal, Indoor and Mobile Radio Communications, PIMRC 2012, pp. 1565-1570.

[9] J.A. Bazerque and G. B. Giannakis, "Distributed Spectrum Sensing for Cognitive Radio Networks by Exploiting Sparsity," IEEE Transactions on Signal Processing, vol. 58, no. 3, pp. 1847-1862, 2010.

[10] M. Melvasalo, J. Lunden, V.Koivunen, "Spectrum Maps for Cognition and Co-Existence o Communication and Radar Systems," in 2016 Conference Record of the 50th Asilomar Conference on Signals, Systems and Computers, Nov 2016.

[11] H. Hentila, J. Oksanen, and V. Koivunen, "Potential field based scheduling in cognitive radio networks," in 2016 IEEE 17th International Workshop on Signal Processing Advances in Wireless Communications (SPAWC), pp. 1-6, July 2016.

[12] S. Chaudhari and M. Kosunen and S. Mäkinen and J. Oksanen and M. Laatta and J. Ojaniemi and V. Koivunen and J. Ryynänen and M. Valkama, "Performance Evaluation of Cyclostationary-Based Cooperative Sensing Using Field Measurements," IEEE Transactions on Vehicular Technology, vol. 65, pp. 1982-1997, April 2016. 